\title{
Channel Classification with Hidden Markov Models in Mobile Networks
}

\author{
By Rafiaa Boujbel ${ }^{*}$
}

In telecommunication networks, Key Performance Indicators (KPIs) are monitored to ensure higher Quality of Service (QoS) in communication networks. With the significant increase of data traffic on the mobile network, a detailed analysis of the transmission quality is becoming increasingly important. Existing classification approaches are widely considered to classify network traffic and not the channel in itself. The aim of this work is to implement a channel estimation tool based on hidden Markov models that is able to determine transmission channel characteristics in mobile radio receivers.

\section{Introduction}

In the field of telecommunication engineering, the term channel refers to the medium between a transmitter and a receiver. The characteristics of wireless signal changes as it travels from the transmitter to the receiver. These characteristics depend upon the distance between the two parts, the signal path and the environment around the path. A channel model, which is a model of the medium between the two parts, is used to obtain the profile of the received signal from the transmitted signal. Due to the enrichment of services and data traffic on mobile networks (e.g. downloads, videos in high resolution), channel classification is becoming crucial to ensure a favorable network quality for users (Yin et al., 2012).

On the one hand, channel classification is widely associated to Global Systems Mobile (GSM) and wireless. On the other hand, existing classification approaches are based on network traffic and not on radio channel itself.

The focus of this work is to combine channel classification with new generation mobile networks. This classification is based on hidden Markov models (HMMs) (Güzelarslan et al., 2011) which are widely used and accepted in the field of digital communication. From the receiver side, a channel classification is useful for giving an overview of the channel conditions. The idea behind is to provide operators with channel estimation tool that has an easy access. Therefore this tool investigates and evaluates the quality and the characteristics of the transmission channel so as to assure a good communication network.

*PhD Student, Munich University of Applied Sciences, Germany. 


\section{Approach}

Consider the case of Single-Input Single-Output (SISO), packets are created and sent in a central network unit (e.g. Operation and Maintenance Center (OMC)). In the mobile radio channel, signals are disrupted and delayed depending on the characteristics of the channel. Due to transmission error, data packets are retransmitted and resulted in delays in the corresponding Internet Protocol (IP) packets. These delays depend on several parameters such as the packet length, the type of packet error and the used error correction mechanisms (Gessner, 2011). The evaluation of the measured jitter of the IP packets and the packet loss lead to estimate the occurred packet error in lower layer. As it is shown in figure 1, sequence errors are generated from jitter. Received binary data determines the corresponding conversion in which "0" indicates an accurate and "1" an erroneous transmission.

Figure 1. Schematic Diagram of Generating Sequence Errors

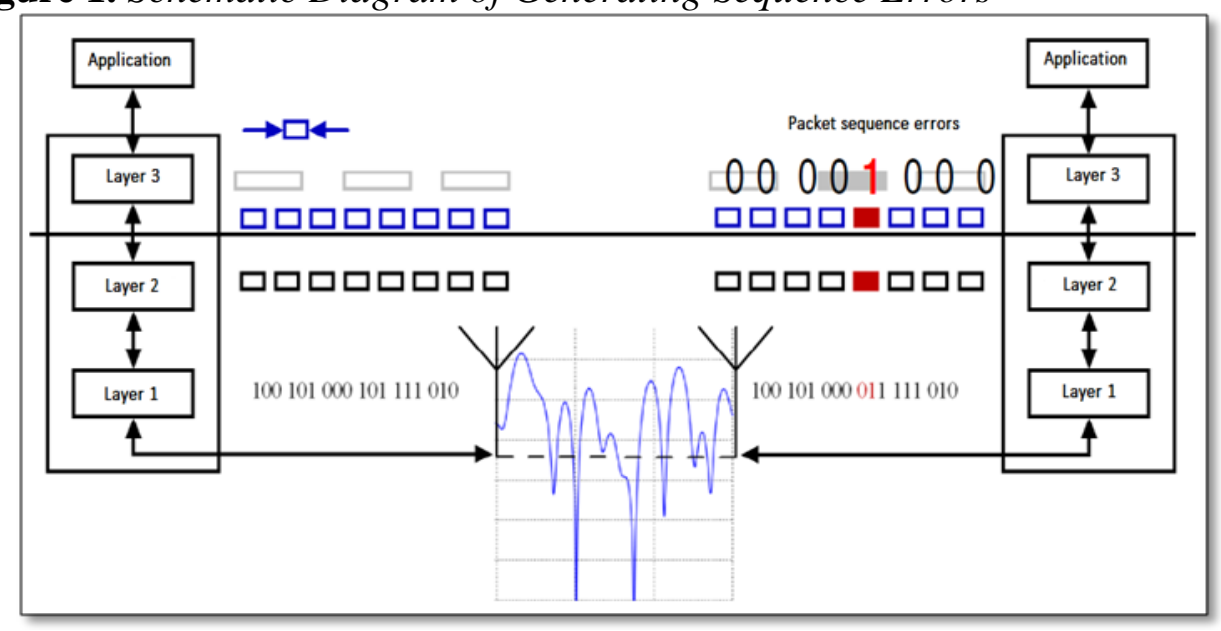

The functional blocks shown in figure 2 are the subject of this work, namely the channel models database and the channel estimator.

Figure 2. Functional Model of Channel Classification in Mobile Networks

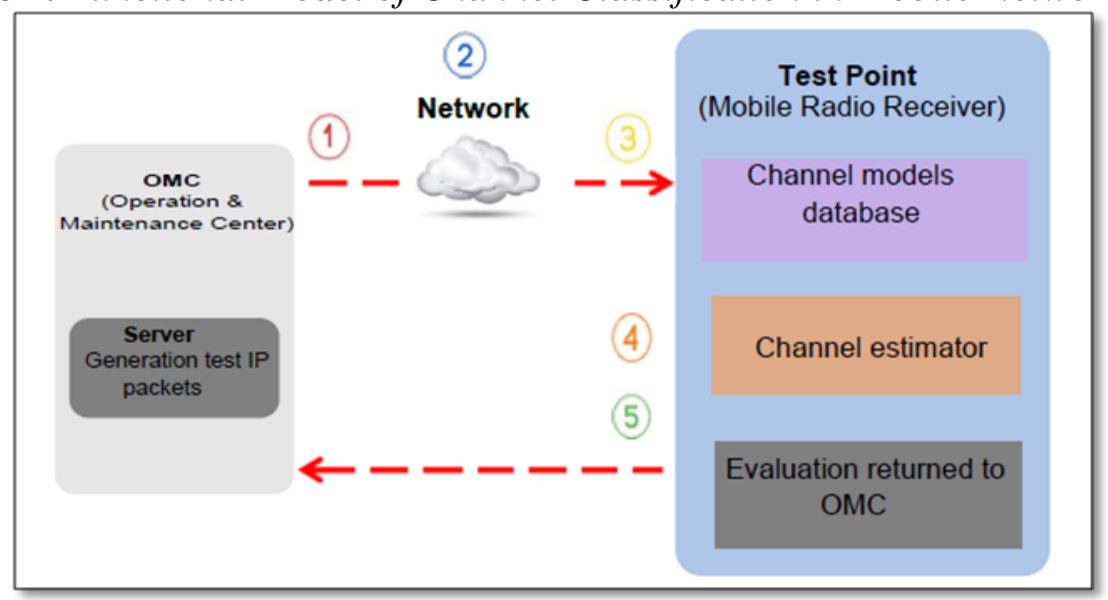




\section{Channel Models Database}

Channel models database is a database of models and precisely of HMMs. These models have been provided from a radio channel simulator on the basis of known channels. To simulate the external environment, a wireless channel emulator is used to emulate wideband radio channel characteristics such as fading profiles which are defined in the $3^{\text {rd }}$ Generation Partnership Project (3GPP) standard.

Considering the case of Long Term Evolution (LTE) network, these fading profiles are divided in low fading namely Extended Pedestrian A model (EPA5), medium fading namely Extended Vehicular A model (EVA5/EVA70) and high fading namely Extended Typical Urban model (ETU70/ETU300) ${ }^{1}$.

These models or HMMs are built specially from sequence errors. Sequence errors are generated from Acknowledgement (ACK) and NegativeAcknowledgement (NACK) protocol message. ACK and NACK are collected via a reliable tool and sequence errors are generated as follow: in case of erroneous packet, a NACK is received and converted to error "1". In case of error-free packet, an ACK is received and converted to " 0 ".

\section{Channel Estimator}

The inputs of the channel estimator are two models and the main classification criteria are the inter-packet delay and the turnaround time. The first model is a HMM already stored in the channel models database. The second model is a HMM generated from the measured signal. In this case, sequence errors are generated from inter-packet delay. When the inter-packet delay is higher than the turnaround time then an error is considered and a " 1 " is generated. When the inter-packet delay is lower than this turnaround time then an error-free is considered and a " 0 " is generated.

The turnaround time consists of the delay between the arrival of a packet at a node and the beginning of its response. It is chosen as a threshold since this time is considered as a criterion for error-free or error packet delivery in mobile networks. For example, LTE standard takes advantage of Adaptive Modulation and Coding (AMC) in addition to the Hybrid Automatic Repeat Request (HARQ) process. This is critical to minimize the turnaround time which is reduced to $8 \mathrm{~ms}$ (Stambaugh, 2008) comparing to previous mobile networks. A detailed explanation of link turnaround time is given in (Johnson at al., 2003).

The channel estimator consists of the metric Kullback-Leibler Divergence (KLD) which is defined in references (Sahraein \& Yoon, 2011, Zhen-Hua \& Li-Rong, 2012). The KLD is used to compare the similarity between these two models described above. Specifically, it is a measure of the information lost when one probability or model is used to approximate the other one. The output of the KLD algorithm is a distance (real number). Smaller is this distance; bigger is the similarity between the models.

${ }^{1}$ ETSI TS 136 104. 2011. LTE; Evolved Universal Terrestrial Radio Access (E-UTRA);Base Station (BS) radio transmission and reception. 3GPP TS 36.104 version 10.2.0 Release 10, 2011 
The classification process of the channel estimator is illustrated in figure 3 . The estimated channel is the result of this classification which is sent back to the OMC.

Figure 3. Classification Process

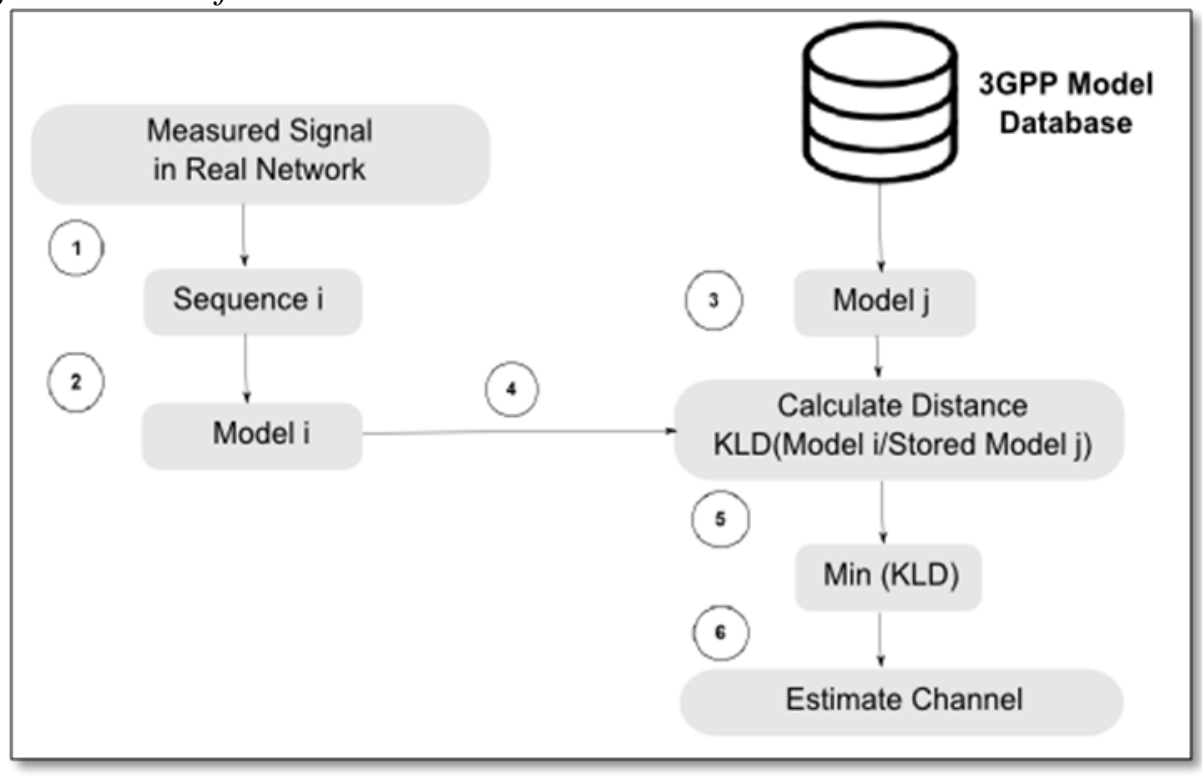

\section{Results}

In order to demonstrate the usefulness of this approach, a wide range of different input models are applied. A numerical example of the classification process is demonstrated by using three standardized LTE radio channel states (3GPP Test EPA5, EVA70 and ETU300, described in ${ }^{1}$ ).

As aforementioned, a measured sequence is compared with a set of error models stored in a database which represent the different radio channel states.

The challenge of the proposed approach is accurately predicting the fading profile used thereby the quality of the channel.

Relying on Kullback-Leibler Divergence, table 1 lists the results of the calculated distances and shows a clear classification of the actual channel: The shortest distance is associated with the most likely radio channel state.

The predicted channel corresponds to the smallest KLD between the measured sequence and models in database. Once the channel is predicted, channel quality and characteristics are obviously known by means of used fading profile. This ensures that channel classification gets visibility as well as channel management by the operator.

${ }^{1}$ ETSI TS 136 104. 2011. LTE; Evolved Universal Terrestrial Radio Access (E-UTRA);Base Station (BS) radio transmission and reception. 3GPP TS 36.104 version 10.2.0 Release 10, 2011 
Table 1. Classification Results for Three Measured Sequences According to 3GPP LTE Standardized Test Cases with Sequence Length T=10, $500 \mathrm{Kbit} / \mathrm{s}$, and $S N R=20 \mathrm{~dB}$

\begin{tabular}{cccccc}
\hline Measured sequences & \multicolumn{5}{c}{ Predicted Model } \\
& EPA5 & EVA5 & EVA70 & ETU70 & ETU300 \\
Sequence 1- EPA5 & $\mathbf{0 . 0 0 3}$ & 0.01 & 0.48 & 0.51 & 0.7 \\
Sequence 2 - EVA70 & 0.265 & 0.19 & $\mathbf{0 . 0 5}$ & 0.437 & 0.56 \\
Sequence 3 - ETU300 & 0.682 & 0.416 & 0.203 & 0.109 & $\mathbf{0 . 0 1 8}$ \\
\hline
\end{tabular}

However, depending on the technology used, models in database referred to the different fading profiles described in the 3GPP standards. The similarity between models is calculated in the same way. Only the turnaround time is varied from technology to another one which is considered to generate sequence errors.

\section{Conclusions and Outlook}

Network traffic classification is the basis of numerous network applications. The classification method used in this work is based on HMM. Experimental results indicate that the combination of channel characteristics and statistical models lead to precise model and achieve higher classification accuracy.

As a continuation of this work, a clustered database can help to detect similar models by speeding up the classification and reducing the number of HMMs.

However, an investigation of Multiple Input Multiple Output (MIMO) channel classification is becoming crucial. The MIMO technology is promising to be one of the key techniques for wireless communications beyond $3 \mathrm{G}$, for its high spectrum efficiency and reliability.

Therefore, extracting jitter values from the received signal without expensive measurement devices and adapting results found to the MIMO technology are the key point for future work.

\section{Acknowledgments}

This project is promoted by the Bavarian Ministry of Economics, Infrastructure, Transport and Technology and it is performed in cooperation with Keynote-SIGOS GmbH. 
Vol. 2, No. $1 \quad$ Boujbel: Channel Classification with Hidden Markov Models...

\section{References}

Gessner, C. 2011. A concise introduction to LTE and its measurement requirements. Rohde \& Schwarz, Germany, 2011, pp. 83.

Güzelarslan, B., Dippold, M. and Paul, M. 2011. Efficient and automatized error pattern modelling with hidden Markov models in digital communication. AEUE, 2011, pp. 417-424.

Johnson, E.E., Balakrishnan. M. and Tang, Z. 2003. Impact of turnaround time on wireless MAC protocols. MILCOM '03 IEEE, 2003, pp. 375-381.

Sahraein, M. and Yoon, B. 2011. A Novel Low-Complexity HMM Similarity Measure. IEEE, 2011, pp. 87-90.

Stambaugh, M. 2008. HARQ Process Boosts LTE Communications. Mobile Dev Design, 2008.

Yin, C., Li, S. and Li, Q. 2012. Network traffic classification via HMM under the guidance of syntactic structure. ELSEVIER, 2012, pp. 1814-1825.

Zhen-Hua, L. and Li-Rong, D. 2012. Minimum Kullback-Leibler Divergence Parameter Generation for HMM-Based Speech Synthesis. IEEE, 2012, pp. 14951497. 